\title{
Anticholinergic drugs and risk of dementia: case-control study
}

\section{(ㄷ) $\mathbb{( 1 )} \Theta$ OPEN ACCESS}

An error occurred in this paper by Richardson and colleagues (BMJ 2018;361:k1315, doi:10.1136/bmj.k1315, published 25

April 2018). The corresponding author's email address should be kathryn.richardson@uea.ac.uk. The paper and PDF will be updated in due course. 